\title{
Introducing Wearable Force Sensors in Martial Arts
}

\author{
Sensors can offer accurate and transparent judging in sports, but as this \\ discussion of the SensorHogu system for taekwondo shows, introducing \\ them raises complicated issues.
}

Ed H. Chi

Palo Alto Research Center echnology has long been altering sports, from the introduction of corked baseball bats to novel tennis rackets to long-flight golf balls and swimsuits made with low-drag material. However, the acceptance of technology in sports has typically been littered with controversy. This is in line with prior experiences in introducing new technologies: it's a complicated process that raises many issues. So, although introducing sensor technology in sports can help promote fairness and spectator enjoyment, a resistance to change exists, partly because such technology also tends to alter player behavior. To succeed, the introduction of ubiquitous technology in sports equipment must therefore account for social acceptance issues, including the players' perception of fairness and rule changes.

In a collaborative effort, the Stanford Taekwondo Program, the Palo Alto Research Center, and Impact Measurement recently developed and introduced sensor technology for the unforgiving environment of the martial arts sparring ring. Our system, called SensorHogu, uses piezoelectric force sensors on body protectors to help taekwondo judges and referees score tournament matches. ${ }^{1}$ We have two objectives for the technology: it should support the judges in accurately scoring the sparring matches, and it should merge with and minimize changes to existing equipment and thus blend into the activity's background.

Introducing SensorHogu to tournament players and judges raised many challenges. First, the system must work technologically without significantly affecting the play of the game. Second, to accept it, the players must perceive the technology as fair and unobtrusive. Third, judges must feel comfortable converting to a new scoring system. Finally, to accommodate the technology's use, rule changes are required. We are currently working with the World Taekwondo Federation (WTF) and United States Taekwondo Union (USTU) to validate and certify the equipment for tournament adoption.

Here, I chronicle our experiences and compare them with technology adoption issues in other sports. To evaluate technology acceptance, we use a framework developed to evaluate ubiquitous computing applications. ${ }^{2}$

\section{SensorHogu: Technological challenges}

Taekwondo has enjoyed enormous popularity for decades and was inducted as an official Olympic sport at the 2000 Sydney Games. The sport's popularity has created increasing pressure to ensure fairness in judging and to make it more spectator friendly. This pressure resulted in several rule changes ${ }^{3}$ and an openness to using technology to ameliorate problems inherent in judging a match. The utmost challenge to accurate 


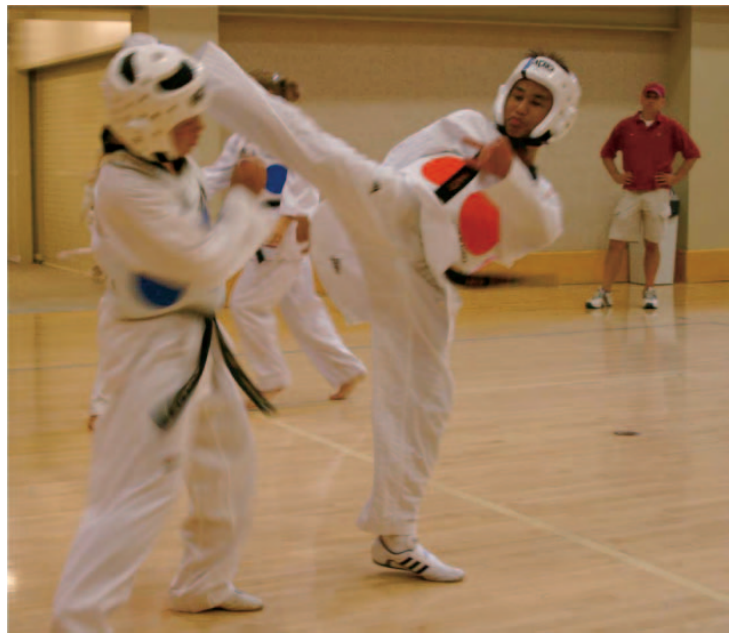

(a)

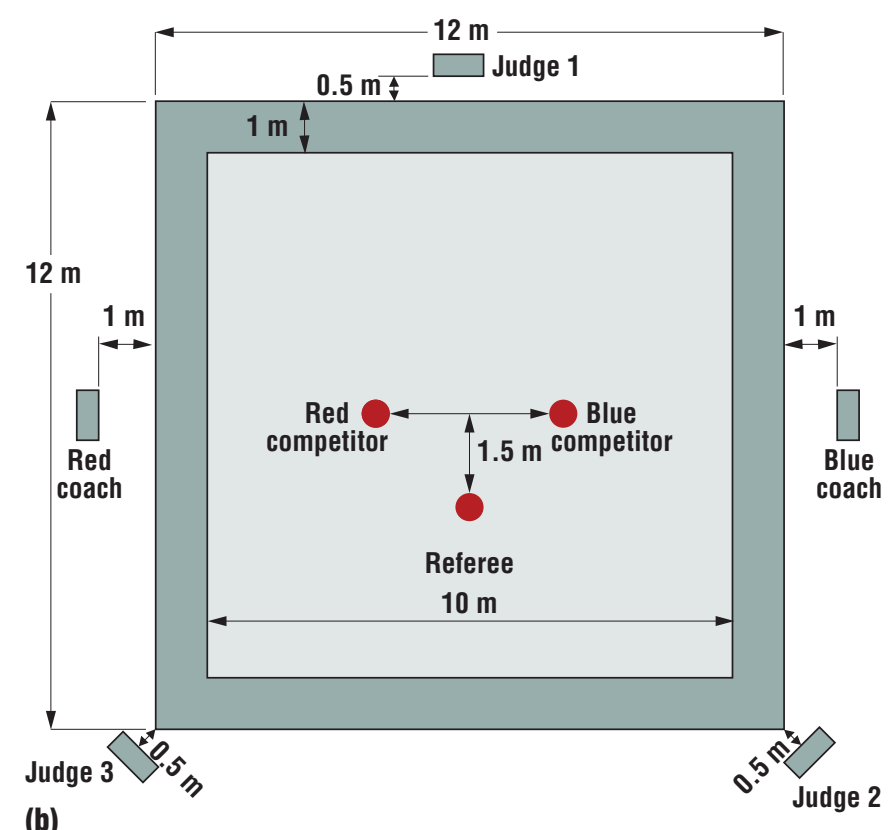

(b)

Figure 1. Taekwondo basics. (a) Two players match up with each other, one wearing a blue body protector and the other wearing red. (b) A standard competition ring, which includes three judges and a referee. The one-meter attention boundary helps the players orient themselves.

scoring is the subjective judgment of what constitutes a valid scoring body kick. According to current rules, a scoring kick must be delivered "accurately and powerfully to the legal scoring area of the body." 3 The subjective nature of this judging criteria has been a major impediment to the sport's development, sometimes resulting in accusations of biased judges favoring players from certain countries (for examples, see the news stories at www.indiavarta.com/olympics/ newHeadlines.asp?cat=Taekwondo).

As figure $1 \mathrm{a}$ shows, taekwondo is a full-contact sport that uses both head and body protectors. Figure $1 \mathrm{~b}$ shows the sport's sparring set up: two competitors (one in a blue body protector, the other in red) move around a square, 12-meter-wide padded mat. Three judges score the hits and a referee conducts the match. As the figure shows, the three judges are placed around the mat in a triangular shape and currently use wired handsets to score the points.

\section{System context and overview}

Figure 2 shows the relationships among the SensorHogu devices. A single base station connects to a laptop, three judges' scoring handsets, and two TrueScore SensorHogu wireless body protectors ("hogu" is the Korean word for "body protector"). SensorHogu uses piezoelectric sensors to detect the amount of force delivered to a competitor's body protector and wirelessly transmits this signal to a computer that scores and displays the point. Our hope is that the force sensors will help judges achieve greater accuracy and help eliminate scoring controversy.

Figure 3 shows the body protector with its wireless transmitter on the upper left shoulder. For the force sensor, we needed something low cost, low powered, and rugged. We didn't consider using accelerometers because we wanted to directly measure the impacting force. The most common dynamic force detector is the piezoelectric sensor. Piezo sensors' stiffness and strength makes them particularly suitable in harsh environments such as a protective suit. In our application, we mounted a single long piece of piezoelectric sensor onto a plastic backing and inserted it into the center of a WTF-approved body protector. We didn't instrument the head and facial area because a mask would obscure the player's vision and facial attacks are easy for judges to score.

The SensorHogu handsets connect wirelessly to a single base station. Each judge has two handsets: the left-hand handset scores the red player (see figure 4) and the right handset scores the blue player. As the figure shows, each handset has a trigger button to score one point for a body hit, and a side button to score two points for a head blow. Force sensors on the body protector must coordinate with the scoring handset, which complicated the system design. According to existing rules for electronic scoring, at least two judges must press the same handset buttons within a one-second window for the point to score. 
Figure 2. SensorHogu system devices. The judges' scores and the SensorHogu data feed into the base station, which connects to a computer that keeps track of scoring according to the rules and displays the match's progress.

\section{Design goals}

We set several design goals when developing the SensorHogu system. First, to facilitate ease of use, we developed a completely wireless system. The standard foilfencing scoring system tethers competitors. ${ }^{4}$ That system, which has been used for decades, is based on electrical switches that complete a circuit when the switches touch a metal vest. Because the tethered setup requires linear fencing forms, it has restricted the sport's natural development. Essentially, human activity has been modified to suit the available technology. With SensorHogu, our goal was not only to transmit contact wirelessly to facilitate ease of use but also to accurately measure the amount of force applied.

Second, our system functions well in real time. Multiple signals from each body protector are transmitted at about the same time, and the system interprets these signals according to the rules. Third, the overall system meets several robustness criteria to withstand an extremely hostile environment. Not only is it small and secure, but the system can resist physical abuse and potential radio interference.

Finally, our system is modular, low cost, and low powered. Both the hogu and transmitter units can be individually replaced, and both can run for up to four days of competition on two AAA-sized batteries.

\section{Gaining trust}

Our next step was to see how well our SensorHogu system worked in practice.

Figure 3. The SensorHogu body protector. The wireless transmitter is stored in a protective pouch on the left shoulder.

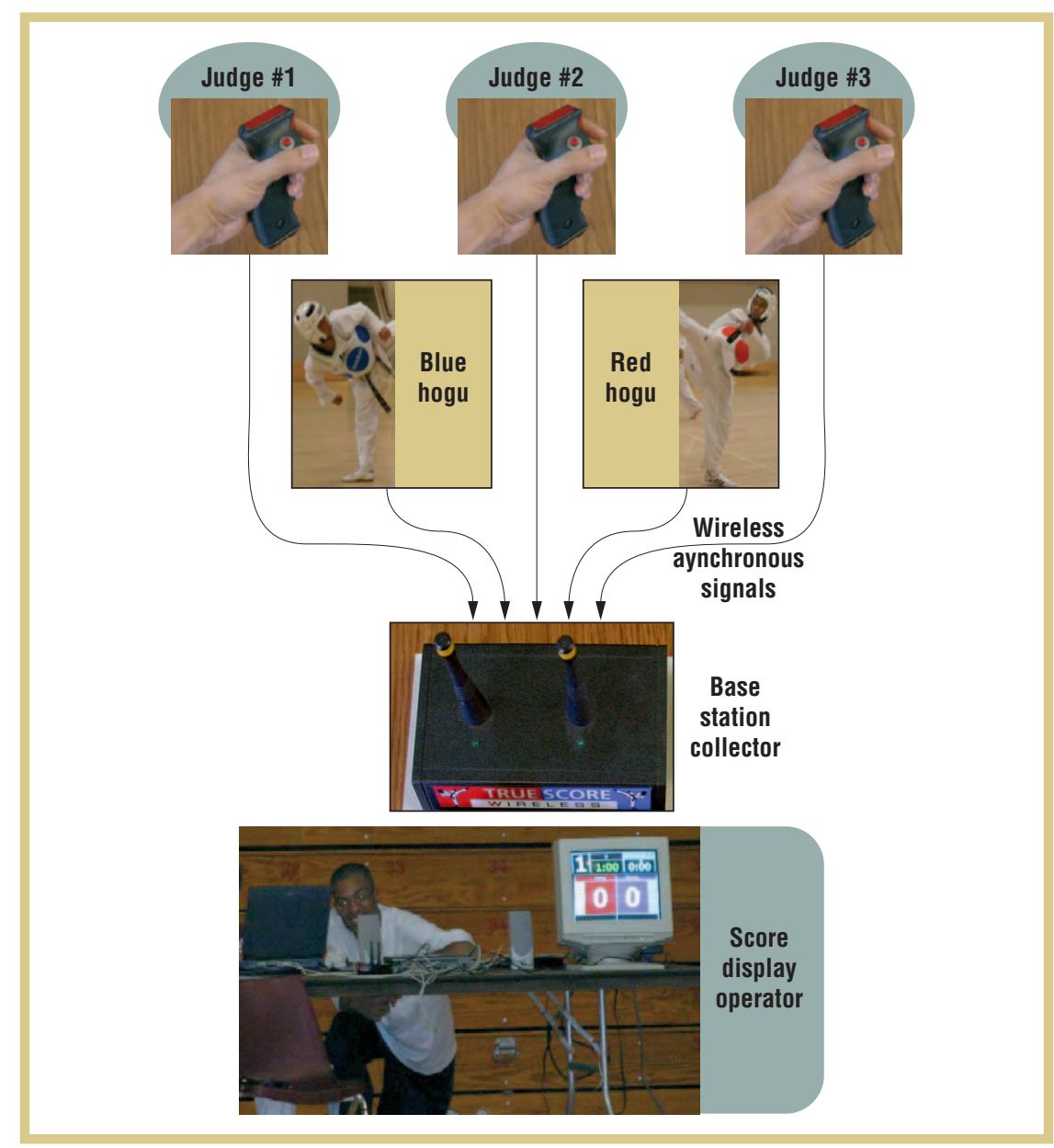

We used two strategies for gaining the trust of players and judges. First, we tested the system with real users and published the results for examination.

Second, we performed live demonstrations at major tournaments.

Judging whether a hit scores a point depends on a variety of factors. Judges

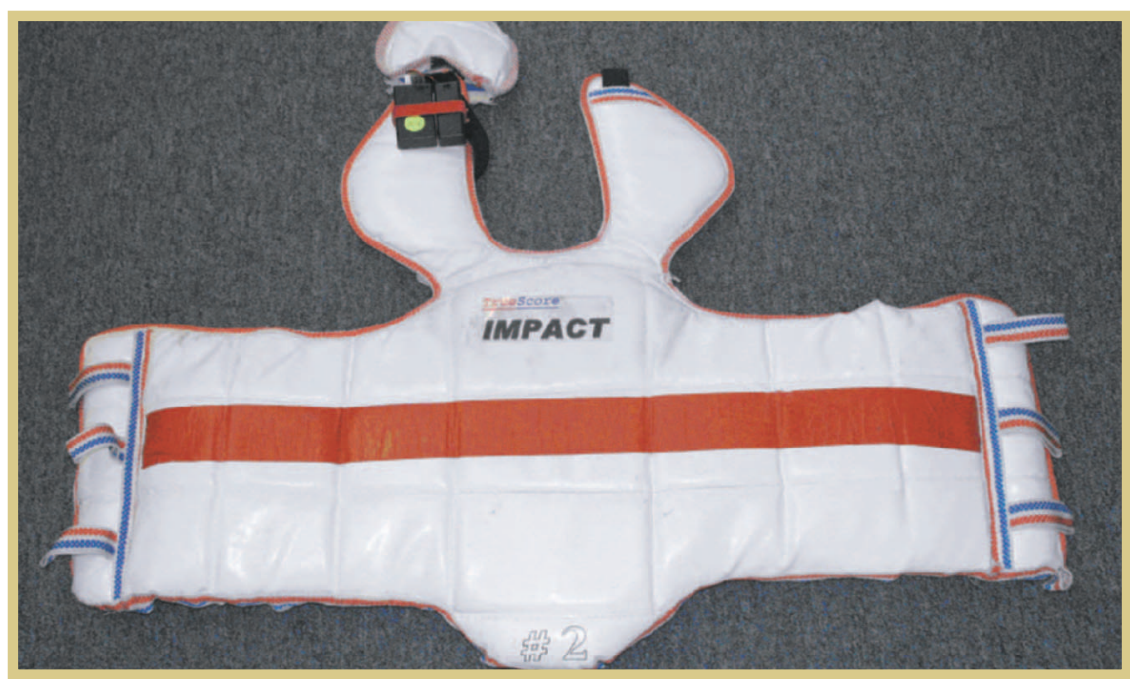




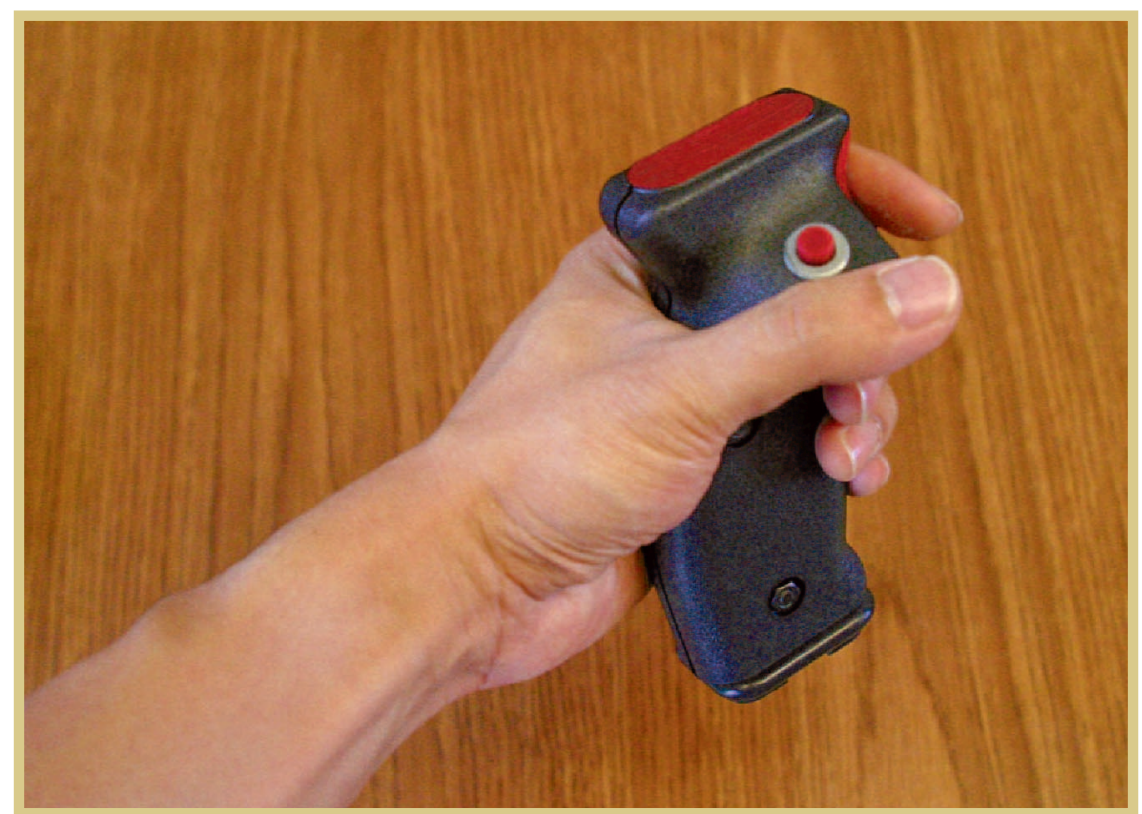

modify their criterion for a point according to the weight division, gender, and type of kicking technique used. There is no easy way to standardize judges' opinions on which kicks are delivered "accurately and powerfully." The resulting inconsistency in judging has eroded some people's confidence and trust, and it was one of the major reasons we developed SensorHogu.

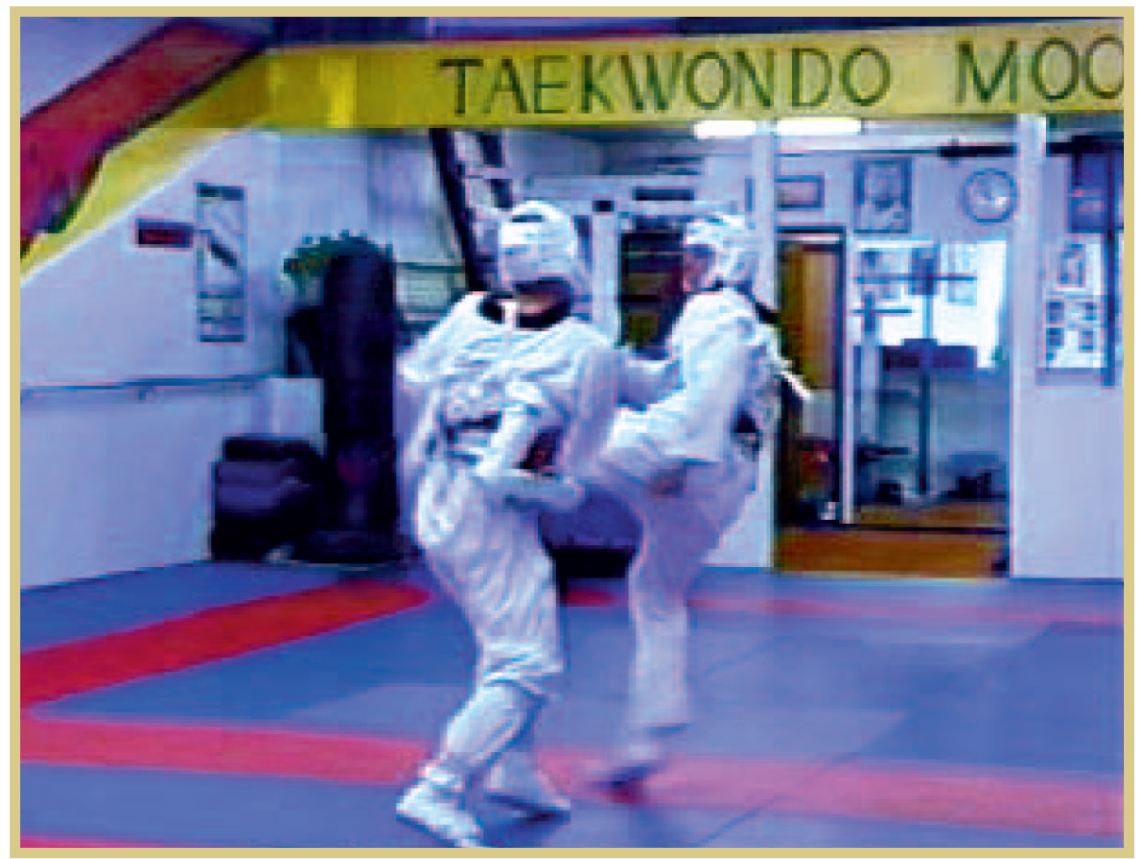

Figure 4. A judge's handset. Because competitions often run for an entire day, the devices are ergonomically designed to eliminate hand fatigue.

we also published live footage of the system in action, and independent judges analyzed the videotapes in slow motion. For example, an analysis of the action in figure 5 shows that a very fast kick might appear to be blocked by the arm, but it actually slipped underneath the elbow; our system scored these cases accurately.

We demonstrated the judges' scoring handsets starting at local tournaments, then gradually introduced these handsets locally, nationally, and internationally. Among the recent tournaments using wireless scoring handsets are the US Taekwondo Union National Championships, US Junior Olympics, and World University Championships. We also recently demonstrated the whole SensorHogu scoring system at the Stanford Open 2004 tournament.

Systems that play such potentially controversial roles in determining match outcomes must be proven valid to players and judges in a variety of ways. Even in the large taekwondo community, wordof-mouth reputation is extremely important to gaining the trust of players and judges.

Certain details of our design played a crucial role in forming community opinion. For example, our handset grip is based on industrially designed joystick grips that reduce fatigue during prolonged use. Judges really appreciated these, as some of the earlier electronic scoring systems used cylindrical plastic handles. Also, players appreciated that our new chest protectors have the same look-and-feel as their normal gear, with few modifications. We also ensured that

Figure 5. Screenshots from the sparring test video. Because of the kicks' speed, it would have been difficult for judges to clearly see that apparently blocked kicks actually hit the body. 
TABLE 1

Evaluating various sports technologies using a ubiquitous computing evaluation framework. The final column is our initial evaluation of SensorHogu.

\begin{tabular}{|l|l|l|l|l|}
\hline Metric & $\begin{array}{l}\text { Bowling: } \\
\text { Foul-line detector }\end{array}$ & $\begin{array}{l}\text { Tennis: } \\
\text { Cyclops }\end{array}$ & $\begin{array}{l}\text { Baseball: } \\
\text { QuesTec }\end{array}$ & $\begin{array}{l}\text { Taekwondo: } \\
\text { SensorHogu }\end{array}$ \\
\hline $\begin{array}{l}\text { Adoption rate/value } \\
\text { Accuracy }\end{array}$ & Good & Fair & Fair & Too early to tell \\
\hline $\begin{array}{l}\text { Predictability } \\
\text { Awareness of capability }\end{array}$ & Good & Good & Good & Good \\
\hline $\begin{array}{l}\text { Minimal distraction } \\
\text { Minimal behavior changes }\end{array}$ & Good & Good & Good & Good \\
\hline Social acceptance & Good & Good & Good & Good (for players and judges; \\
\hline & Good & Fair & Fair & Goo early to tell for spectators) \\
\hline
\end{tabular}

the system's scoring beep isn't distracting to the players.

To use the SensorHogu in tournament matches requires rule changes. In May 2005, WTF changed the rules to specify two rather than four judges when electronic hogu technology is in use. In fact, WTF had changed the rules in anticipation of the new technology and had called for possible technology partners. We will be demonstrating our technology to WTF taekwondo officials in Seoul in July 2005, in hopes of becoming the first electronic hogu system on the market. Generally, organizational changes are extremely difficult to push forward, however, particularly when they involve the rule changes required to allow electronic scoring equipment. Fortunately, there is pressure from the Olympic committee to introduce new scoring technology in taekwondo, and we hope to introduce the system in the 2008 Olympics.

\section{Evaluation framework for sports technology}

To evaluate SensorHogu and compare it to other marketed sports technologies, we used an evaluation framework for ubiquitous computing applications developed by Jean Scholtz and Sunny Consolvo. ${ }^{2}$ They suggested nine critical

Figure 6. The bowling foul-line detector is widely used in bowling alleys around the world. evaluation areas for understanding a ubiquitous computing technology: attention, adoption, trust, conceptual models, interaction, invisibility, impact, appeal, and application robustness. They also suggested a set of metrics for evaluating each area (34 metrics in all).

After reviewing the SensorHogu, Dario Salvucci of Drexel University applied the Scholtz and Consolvo metrics to various sports technologies and roughly estimated how different technologies performed. I discussed these ideas with him in depth at the 2004 Human-Computer Interaction Consortium Symposium in Frasier, Colorado. Based on these discussions and his unpublished slides, I offer here a subset of the applicable metrics as applied to three existing sport technologies. I then evaluate the SensorHogu system using the same metrics. Table 1 summarizes how SensorHogu and systems in bowling, tennis, and baseball faired in relation to the simplified evaluation framework.

\section{Bowling: Foul-line detector}

In bowling, when a player steps over the foul line, a simple system detects the infraction and issues a beep (see figure 6). The system also automatically counts the pins and scores the game. (A bit of trivia: as a new startup in Silicon Valley, Hewlett Packard tried to sell a bowling foul-line indicator as far back as 1938.)

This foul-line technology is a poster child for a sensor system's successful

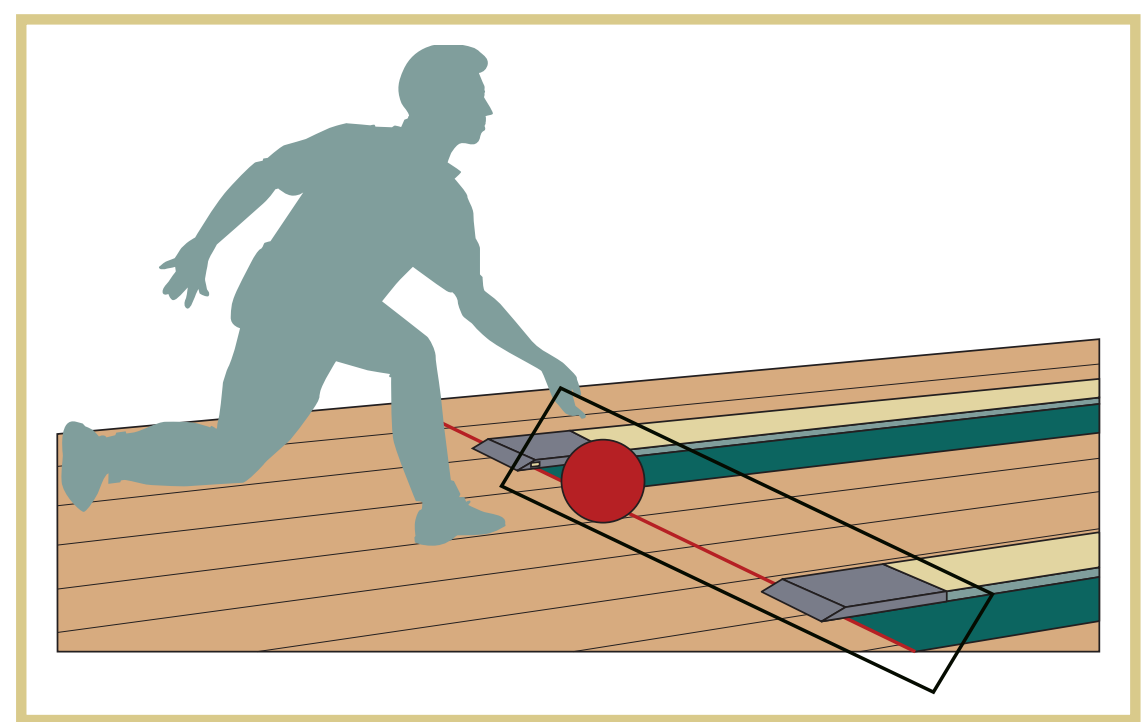




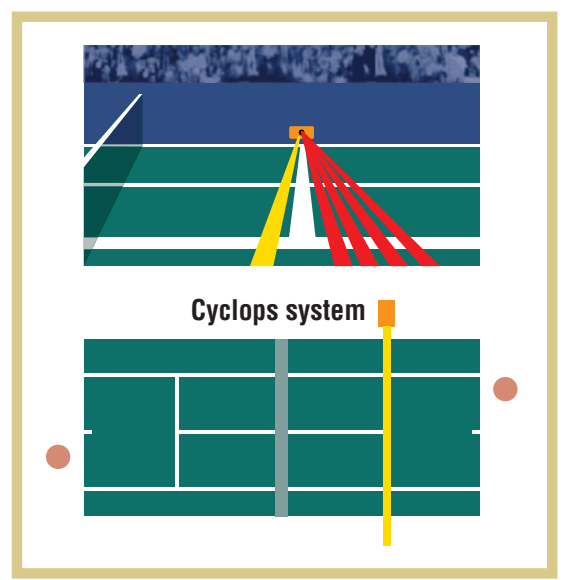

Figure 7. The Cyclops system. When players serve, the technology calls "long" for a single line on the tennis court.

adoption within a sport. The systems are everywhere, and they're extremely accurate. Players understand how they work and can easily predict system behavior. Because the beep occurs after players have released their balls, the system is not distracting and requires no behavioral changes. Socially, the systems are as much part of the sport as any other part of the bowling alley.

Figure 8. The QuesTec video analysis system for baseball. (figure courtesy of InTech magazine/ISA)

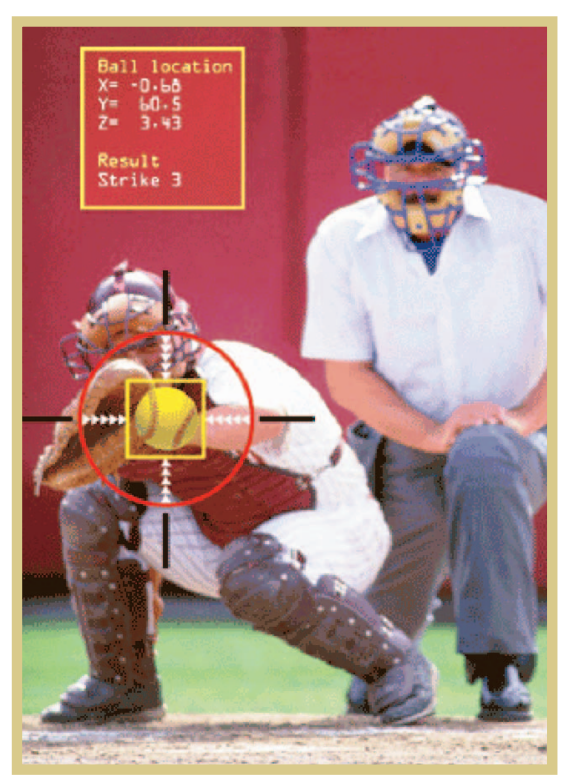

\section{Tennis: Auto serve-line detector}

Major tennis tournaments have used a system with functionality similar to the bowling technology since the 1990s. Cyclops detects whether serves are "in" or "long" (see figure 7). It doesn't detect wide serves, and referees can overrule its calls. Cyclops created quite a stir when it was first introduced but eventually won reluctant acceptance.

Cyclops systems are highly accurate and consistent in their detections, and how they work is easily understood. Despite sharing these characteristics with the bowling foul-line detector, Cyclops has achieved only limited adoption. While it doesn't directly change the serve or the receiving player's strategy, the system is distracting. The beep occurs just before the receiving player is about to strike the ball. This breaks both players' concentration and focus and, therefore, its social acceptance has been limited. To remedy this, researchers must further study the beep's ergonomics and its effect on players to perhaps better optimize its timing. The beep might be less distracting, for example, if it occurred after the receiving player returned the ball.

\section{Baseball: Video analysis of balls and strikes}

The QuesTec Umpire Information System offers video analysis of balls and strikes in baseball (see figure 8). In the US, a huge dispute over its use put the system at the center of labor negotiations between Major League Baseball and its umpires. This dispute wasn't settled until recently, ${ }^{5}$ and the resolution is still subject to ratification. Thirteen of the 30 major league stadiums have installed QuesTec, and baseball officials have used its data to grade umpire performance. As a reporter noted in a New York Times article, "If an umpire's calls disagree with the computer's more than 10 percent of the time, his performance will be considered substandard and pos- sibly held against him in future promotion considerations and when lucrative post-season assignments are made. The umpires are, naturally, freaked out by QuesTec."6

Players are just as unhappy about the system. Arizona Diamondbacks pitcher Curt Schilling was fined for smashing a QuesTec camera after being told umpires are "changing their strike zones to match the machine." Atlanta Braves' pitcher Darren Holmes was quoted as saying that "this system is one of the worst things that has happened in baseball."”

Players and umpires understand how the system works. It has nonetheless proven a major distraction for hitters and pitchers alike. The reason is that QuesTec forced definitive behavioral changes. Umpires had to change their strike zones to conform to QuesTec's zones, which forced pitchers to deliver pitches to the new zones and modify their mix of pitches. In turn, hitters had to adjust to new strike zones and pitching styles. These effects resulted in extremely poor social acceptance of the technology. Thus, despite its fairly accurate and consistent performance, most parks haven't installed the system, creating inconsistencies across the league.

\section{Taekwondo: SensorHogu}

After discovering what happened to the QuesTec system, our development team was naturally concerned because, as with QuesTec, SensorHogu sets a threshold standard that players must meet to earn a score. Although inconsistent and poorly understood, the original standard of a hit being delivered "accurately and powerfully" had been used for a long time and a culture of understanding had been built around it. Players and judges made adjustments based on their personal interpretations of the rules and match situations; inconsistencies became somewhat a part of the game. As with QuesTec, winning Sen- 
sorHogu acceptance required that sports officials not only buy in to it but also believe in our system's accuracy and fairness. Moreover, it was crucial that SensorHogu not force any unwanted behavioral changes on players or judges. Our design mantra was "every design is for the benefit of the players and the fairness of the game."

SensorHogu has yet to be officially introduced and sanctioned. It's therefore too early to discern or predict SensorHogu's adoption rate. As our published results suggest, ${ }^{1}$ the system is consistent and fairly predictable to judges and players, and the sensors are reliable enough to be used for fair play. Also, players and judges easily understand how the system works. In our test matches, players performed as if the system wasn't there and have not yet modified behaviors or strategies while wearing it. So far, players and judges seem to enjoy using the technology and appreciate its added value to the game. Many of these early opinions are based on the system's reputation and inherent reliability. Opinions might change, however, if SensorHogu experiences a high-profile failure, which we are working to prevent. Table 1 summarizes our initial evaluation of the SensorHogu system.

In taekwondo, the audience is a third party and has an indirect stake in this issue. Given this, it might be valuable to display how hard someone's hogu is hit during each encounter. This could add entertainment value and make the opaque culture of scoring thresholds more transparent and easily verifiable. Another uncertainty is that we currently do not know if judges are more likely to score a kick that has a higher degree of difficulty but generates less power than other types of kicks. If this is currently true, SensorHogu's use might alter kick selection and thus change player behavior by making the player favor more powerful kicks.

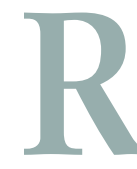
eviewing how technology alters sports raises several broad questions: How do we want technology to augment sports and help referee them? Can we systematically understand when a technol-

\section{REFERENCES}

1. E.H. Chi, J. Song, and G. Corbin, “'Killer App' of Wearable Computing: Wireless Force Sensing Body Protectors for Martial Arts," Proc. 17th Annual ACM Symp. User Interface Software and Technology, ACM Press, 2004, pp. 277-285.

\section{Blindly developing sports technology without}

\section{thinking how it affects players' behavior, judges'}

\section{scoring choices, and spectator perceptions}

\section{is a clear recipe for disaster.}

ogy works well or not? When does a technology start to "ruin" the spirit of a sport?

Researchers have envisioned a variety of application areas for sensors, including everyday environments such as the home or office. Evaluators have studied technology adoption in such areas ${ }^{2}$ but not yet in sports. Sensors offer many possibilities in this area, including their use in

- hockey pucks, to detect when they cross goal lines;

- golf balls, to better track their trajectory; and

- footballs, to better sense the last point of forward momentum.

Adopting sensor technology in sports appears to operate on a similar set of evaluation factors as other areas of ubiquitous computing. Blindly developing sports technology without thinking how it affects players' behavior, judges' scoring choices, and spectator perceptions is a clear recipe for disaster. We hope that our SensorHogu work points to future work necessary in understanding when a ubiquitous computing application in sports has the potential for being a "killer app." $\mathbf{P}$

\section{ACKNOWLEDGMENTS}

I thank Dario Salvucci for his figure ideas and generous discussions on sports technology evaluations, which contributed significantly to this article.
2. J. Scholtz and S. Consolvo, "Toward a Framework for Evaluating Ubiquitous Computing Applications," IEEE Pervasive Computing, vol. 3, no. 2, 2004, pp. 82-88.

3. World Taekwondo Federation, Competition Rules \& Interpretation, 2003.

4. C. Freudenrich, "How Fencing Equipment Works," 2003, www.howstuffworks.com/ fencing-equipment.htm.

5. M. Chass, "Baseball and Umpires Settle Grading Dispute," New York Times, 24 Dec. 2004; www.questec.com/q2001/ spfe_nytimes_122404.htm.

6. H. Lindgren, "The Foolproof Umpire," New York Times, 14 Dec 2003; www. questec.com/q2001/spfe_nytimes_1203.htm.

7. B. Wilson, "Schilling Fined about $\$ 15,000$ for Destroying Questec Camera," Sports Illustrated, 2 June 2003; http://sportsillustrated. cnn.com/baseball/news/2003/06/02/schilling fined_ap.

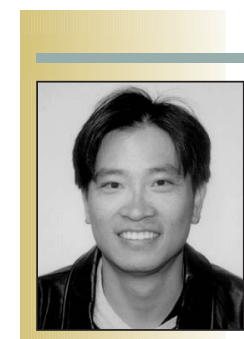

the AUTHOR

Ed H. Chi is a senior research scientist at Palo Alto Research Center's User Interface Research Group. His research interests are in user interface software systems. He received his $\mathrm{PhD}$ in computer science from the University of Minnesota. He is a senior member of the IEEE. Contact him at PARC, 3333 Coyote Hill Rd., Palo Alto, CA 94304; echi@parc.com. 